University of Wollongong

Research Online

Faculty of Engineering - Papers (Archive)

Faculty of Engineering and Information

Sciences

$1-1-2012$

\title{
Charge carrier exchange at chemically modified graphene edges: A density functional theory study
}

Ting Liao

University of Queensland

Chenghua Sun

University of Queensland

Aijun Du

University of Queensland

Ziqi Sun

University of Wollongong, ziqi@uow.edu.au

Denisa Hulicova-Jurcakova

University of Queensland

See next page for additional authors

Follow this and additional works at: https://ro.uow.edu.au/engpapers

Part of the Engineering Commons

https://ro.uow.edu.au/engpapers/4844

\section{Recommended Citation}

Liao, Ting; Sun, Chenghua; Du, Aijun; Sun, Ziqi; Hulicova-Jurcakova, Denisa; and Smith, Sean: Charge carrier exchange at chemically modified graphene edges: A density functional theory study 2012, 8321-8326.

https://ro.uow.edu.au/engpapers/4844

Research Online is the open access institutional repository for the University of Wollongong. For further information contact the UOW Library: research-pubs@uow.edu.au 
Authors

Ting Liao, Chenghua Sun, Aijun Du, Ziqi Sun, Denisa Hulicova-Jurcakova, and Sean Smith 


\title{
Journal of \\ Materials Chemistry

\section{Charge carrier exchange at chemically modified graphene edges: a density functional theory study $\dagger$}

\author{
Ting Liao, ${ }^{a}$ Chenghua Sun, ${ }^{a b}$ Aijun Du, ${ }^{a}$ Ziqi Sun, ${ }^{c}$ Denisa Hulicova-Jurcakova ${ }^{b}$ and Sean Smith ${ }^{* d}$ \\ Received 19th January 2012, Accepted 21st February 2012 \\ DOI: 10.1039/c2jm30387f
}

\begin{abstract}
Heteroatom doping on the edge of graphene may serve as an effective way to tune chemical activity of carbon-based electrodes with respect to charge carrier transfer in an aqueous environment. In a step towards developing mechanistic understanding of this phenomenon, we explore herein mechanisms of proton transfer from aqueous solution to pristine and doped graphene edges utilizing density functional theory. Atomic B-, N-, and O- doped edges as well as the native graphene are examined, displaying varying proton affinities and effective interaction ranges with the $\mathrm{H}_{3} \mathrm{O}^{+}$charge carrier. Our study shows that the doped edges characterized by more dispersive orbitals, namely boron and nitrogen, demonstrate more energetically favourable charge carrier exchange compared with oxygen, which features more localized orbitals. Extended calculations are carried out to examine proton transfer from the hydronium ion in the presence of explicit water, with results indicating that the basic mechanistic features of the simpler model are unchanged.
\end{abstract}

\section{Introduction}

The increasing energy demand has stimulated the rapid growth on discovering novel nanomaterials for improving the performance of sustainable energies. ${ }^{1,2}$ Following broad study and applications of graphene, there has been growing interest in graphene nanoribbons as possible passive and active components in energy generation and storage devices. ${ }^{3-10}$ For carbonbased energy devices in an aqueous environment, the interface between acidic/basic solutions (electrolyte) and graphene nanoribbons therefore constitutes an important model system. ${ }^{6,11-15}$ A complete description of charge carrier transfer in aqueous acidic solution requires minimally an understanding of interactions among hydrated charge carriers, water, and the electrode surface. Proton transport in water involves the migration of a topological defect in the water network via a sequence of proton-transfer reactions. While there has been

${ }^{a}$ Centre for Computational Molecular Science, Australian Institute for Bioengineering and Nanotechnology, University of Queensland, Brisbane QLD 4072, Australia.E-mail: t.liao1@uq.edu.au; c.sun1@uq.edu.au; a. du@uq.edu.au; Fax: +61(7)3346 3992; Tel: +61(7)3346 3972

${ }^{b}$ ARC Centre of Excellence for Functional Nanomaterials, Australian Institute for Bioengineering and Nanotechnology, University of Queensland, Brisbane QLD 4072, Australia. E-mail: d.jurcakova@uq. edu.au; Fax: +61(7)3346 3973; Tel: +61(7)3346 3830

'Institute for Superconducting \& Electronic Materials, University of Wollongong, NSW 2500, Australia. E-mail: ziqi@uow.edu.au; Fax: +61(2) 42215731; Tel: +61(2) 42981479

${ }^{d}$ Centre for Nanophase Materials Sciences, Oak Ridge National Laboratory, Oak Ridge, TN 37831, USA. E-mail: smithsc@ornl.gov; Fax: +1(865)574 1753; Tel: +1(865)574 5081

$\uparrow$ Electronic supplementary information (ESI) available. See DOI: $10.1039 / \mathrm{c} 2 \mathrm{jm} 30387 \mathrm{f}$ much debate over many years as to the best characterization of the topological defect, for the present purposes we couch our discussion in terms of the hydrated proton, $\mathrm{H}_{3} \mathrm{O}^{+}$, while noting that our extended water interface calculations below do not preclude larger clusters.

In carbon nanosheets, edge chemistry plays an important role in the overall material properties, such that understanding the nature of functionalized edges of graphene nanoribbons underpins our ability to rationally design such materials for specific applications. ${ }^{16-19}$ It is of great importance to develop optimized electrode materials based on graphene which combine both the intrinsic high charge carrier conductivity and chemical stability in aqueous solutions. It is widely reported that functionalized graphene nanoribbons can speed/hinder charge carrier exchange and hence affect the overall performance of electrodes used in diverse electrochemical devices. ${ }^{\mathbf{1 8 2 0}}$ For instance, recent work has shown increased charge transfer in nitrified active carbon. ${ }^{21}$ The mechanism is believed to be related to the incorporation of nitrogen ions near the ribbon edge and proton transport between these species and electrolyte or along the edge, but a detailed experimental characterization of the mechanism remains elusive. It is thus of particular importance to understand the interplay between doped edges and hydrogenated charge carriers, since this can influence the structure and stability of the charged bilayers and hence provides a key to tuning charge transfer and improving the electrochemical performance.

What is clearly needed is a mechanistic picture that clearly elaborates charge carrier exchange at the interface. One method for approaching this problem is via ab initio calculation, which incorporates electronic structure explicitly and allows for 
correct description of chemical bond rearrangements. The structure and dynamics of hydrated protons has been studied by many authors by means of calculations at different levels of theory [e.g., ref. 22-27]. In this work, we focus specifically on the mechanism of the charge carrier transfer at graphene nanoribbon edges from a first principle computational point of view, considering both structural and electronic aspects. The paper is organized as follows: we first compute energy profiles and resultant equilibrium structures for charge carrier transfer between the hydronium ion and bare and doped edges of carbon nanoribbons. We then examine the effect of explicit inclusion of water layers on the pathway for charge carrier transfer from hydronium to the ribbon edge. Finally electronic structures are examined to gain further insights into the charge transfer phenomenon.

\section{Computational methods}

Our first principles calculations are based on density-functional theory (DFT), as implemented in the PWSCF package. ${ }^{28}$ The PBE (Perdew-Burke-Ernzerhof) exchange-correlation functional ${ }^{29}$ within the generalized gradient approximation (GGA) has been used. The electron-ion interactions are described with ultrasoft pseudopotentials, ${ }^{30}$ which allow for an accurate treatment with a relatively moderate plane wave cutoff of $40 \mathrm{Ryd}$. The Brillouin zone is sampled using a $k$-point mesh of $7 \times 1 \times 1$ within Monkhorst-Pack scheme ${ }^{31}$ for the nanoribbon structures. We have performed extensive convergence tests on the planewave energy cutoff as well as the number of $k$ points with the criterion that the total energy be converged to $\sim 0.02 \mathrm{eV} /$ atom or better.

Zigzag shaped nanoribbons are particularly interesting as they have localized edge states exhibiting activity. A $(8,3)$ supercell of zigzag shaped graphene nanoribbon is constructed with $\mathrm{H}$-saturated boundaries on both sides except for the examined edge atom with or without dopant, wherein the unit is eight rows of hexagonal rings of carbon atoms (8-ZGNR) and the supercell is three times larger than the unit. We have used a vacuum whose size perpendicular to the nanoribbon surface and on the unsaturated edge of nanoribbon has been fixed to $15 \AA$. We have checked that such supercell size is large enough to avoid spurious interactions between the hydronium and its periodic replicas.

Weak noncovalent hydrogen bonds play a central role in describing the nature of molecular interactions in aqueous environment. However, the accurate description of this interaction remains a major challenge for $a b$ initio electronic structure theories. Density functional theory (DFT), which is widely used in electronic structure calculations, is often inadequate in describing such interactions because of the local or semilocal nature of common exchange-correlation $(x c)$ functionals in widespread use. There are various strategies to fix this deficiency such as $x c$ functional which explicitly account for nonlocal correlation, using maximally localized Wannier functional, ${ }^{32,33}$ dispersion-corrected atom-centered potentials, ${ }^{34,35}$ or damped empirical $C_{6} R^{-6}$ corrections (DFT-D) ${ }^{36,37}$ In this work, we used a London-type empirical correction for hydrogen bond (dispersion interaction) whose main idea of the algorithm consists in adding the $C_{6} R^{-6}$ empirical potential to the exchange-correlation potential, where $R$ is the distance between the atoms in different molecules and $C_{6}$ is the dispersion coefficient.

\section{Results and discussion}

\subsection{Charge carrier transfer dynamics}

The molecular geometry of hydronium $\left(\mathrm{H}_{3} \mathrm{O}^{+}\right)$is trigonal pyramidal (The molecular geometry, lowest manifold of molecular orbitals and related parameters for validation purposes are summarized in ESI $\dagger$ ). Fig. 1a shows the favored $\mathrm{H}_{3} \mathrm{O}^{+}$orientation upon approach to the ribbon edge, which is reasonable given the energetic drive to maximize orbital overlap between the proton $\mathrm{H}^{+} 1 s$ in hydronium ion and $2 p$ states of the edge atom. To determine the critical distances at which the proton transfer occurs, the potential energy of the hydronium interacting with pristine and also doped edges as a function of the separation between the edge site and the oxygen of $\mathrm{H}_{3} \mathrm{O}^{+}$was calculated using constrained optimization. Starting from a large separation at $5.0 \AA$, the separation was incrementally decreased with an averaged interval of $0.5 \AA$ and optimization carried out at each step. The interaction energy at any separation is calculated according to

$$
\mathrm{E}_{\mathrm{int}}=\mathrm{E}_{\mathrm{H} 3 \mathrm{O}+\text {-ribbon }}-\mathrm{E}_{\text {ribbon }}-\mu_{\mathrm{H} 3 \mathrm{O}+}
$$

where $\mathrm{E}_{\mathrm{H} 3 \mathrm{O}+\text {-ribbon }}$ is the total energy of ribbon interacted with hydronium, $\mathrm{E}_{\text {ribbon }}$ is the energy of the bare ribbon with different doping, $\mu_{\mathrm{H} 3 \mathrm{O}+}$ is the total energy of a hydronium ion. Fig. $1 \mathrm{~b}$ shows the interaction energy profiles as a function of the
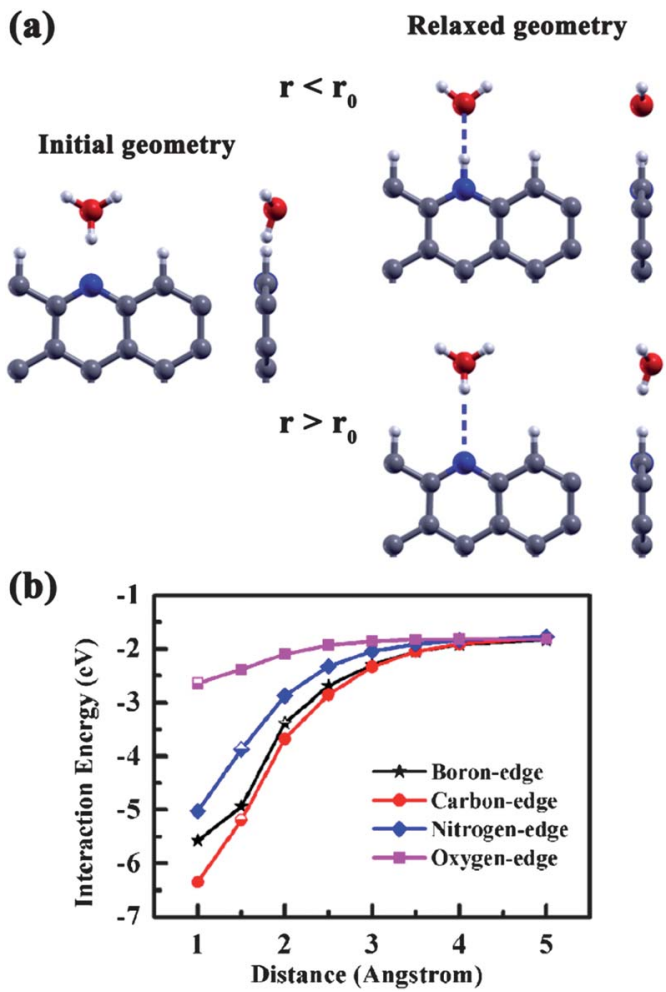

Fig. 1 (a) Initial geometry and equilibrium structures of hydronium interacting with doped edge of grapheme nanoribbons within or beyond critical distances $d_{0}$, only first two carbon atomic layers are shown for simplicity. (b) Relative energy profiles for transferring a proton from hydronium to functionalized GNRs' edge as a function of the distance, $d$, of hydronium from the exposed edge. 
interaction distance, $r$, of hydronium from the exposed edge. Examination of the corresponding structures reveals that there is a definite critical distance $r_{0}$, highlighted by the half-filled symbols in each of the lines of Fig. 1b, at which point the proton relaxes onto the edge site and is separated from the water. This critical distance varies between $1.0 \AA \sim 2.0 \AA$ for the different dopant systems studied. The relaxation geometry of hydronium as it approaches the edge is shown in Fig. 1a. If the hydronium ion separation from the ribbon edge is greater than the critical distance $r_{0}$, i.e. $r>r_{0}$, the final equilibrium geometry ends with the initial geometry almost unchanged, indicating that hydronium does not specifically dissociate. This leads to the formation of a weak hydrogen bond between the hydronium ion and nanoribbon edge with essentially no charge transfer, as one would expect of a physisorption. However, when hydronium ion moves within the critical distance $r_{0}\left(r \leq r_{0}\right)$, the ion cluster is destabilized due to the $\mathrm{H}_{2} \mathrm{O}-\mathrm{H}^{+}$...edge Coulomb interaction and dissociates into $\mathrm{H}^{+}$ion and water molecule. In the successive optimization iterations, the water molecule moves further away from the ribbon edge leaving the leading $\mathrm{H}^{+}$ion bonded to the edge atom. Still, a weak hydrogen bond is formed between dissociated proton and water molecule. The hydronium-ribbon interactions elaborated above lend support to the model of electrochemical proton-transfer reactions in which the rate determining step is when a proton is passed on to the ribbon edge, from a hydrogenated charge carrier in the solution which is in contact with the electrode. The charge carrier transfer reaction can therefore be represented in terms of the following equation,

$$
\mathrm{H}_{3} \mathrm{O}^{+}+\mathrm{GNR} \leftrightarrow \mathrm{H}_{2} \mathrm{O}+\mathrm{GNR}-\mathrm{H}^{+}
$$

Proton transfer from the hydronium to an active ribbon edge is barrierless in all cases studied here. The increasing critical interaction distance $r_{0}$ for $\mathrm{H}_{3} \mathrm{O}^{+}$dissociation on the ribbon edges, specifically, $1.0 \AA$ for $\mathrm{O}, 1.5 \AA$ for $\mathrm{C}$ and $\mathrm{N}$, and $2.0 \AA$ for $\mathrm{B}$, correlates with increasing atomic radius - an indicator of diffuseness of the outer valence orbitals. Concomitant with its effectively longer range interaction with the hydronium ion, transfer of proton charge carriers is apparently optimized with boron terminated edges amongst those considered here. This result gives simple and transparent explanation of the experimental observation that boron-doped active carbon exhibits higher interfacial capacitance than that of boron-free carbon. ${ }^{38}$ Proton transfer from hydronium at the critical distance of $2.0 \AA$ onto a hydrogen-passivated B-doped ribbon edge was also examined: the exchange of proton to the active boron site proceeds analogously in this case (Fig. S2 in ESI).

Interestingly, the interaction energy at the doped edges is in all cases higher than the corresponding one for the pristine ribbon. This difference may originate from the larger lattice elastic relaxation observed in doped ribbon edges. Nevertheless, the calculated large negative interaction energies in all doped cases guarantee an easy transfer of protonic charge carriers to ribbon edge when hydronium is within the effective dissociative region of each edge (i.e., $r \leq r_{0}$ ).

The chemical bond formed between the proton and the edge atom $\left(d_{1}\right.$, see labels in Fig. 2$)$ is shortest in the case of nitrogen. This translates to a stronger bond between nitrogen edge atom
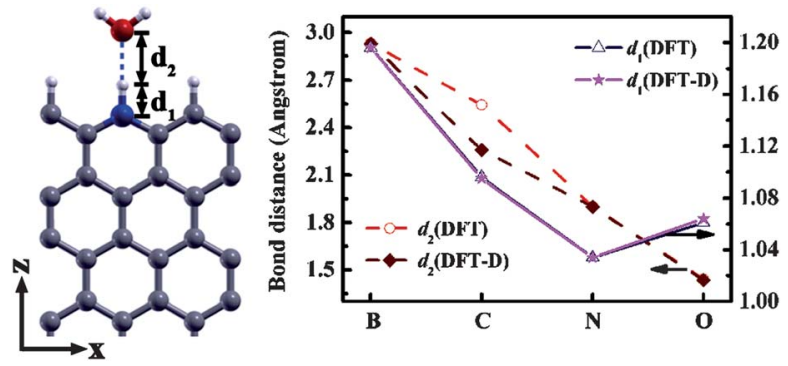

Fig. 2 On the left: supercell of doped zigzag shaped graphene nanoribbon with proton transferred from hydronium to ribbon edge; on the right: atomic bond lengths for different doped ribbon edges as denoted in the left panel.

and proton and, most likely, a greater stability of the proton on the edge and consequently a decreased likelihood of de-protonation or proton transport. The hydrogen bond between the proton and oxygen atom of the water molecule (labeled $d_{2}$ ) is more elongated than the chemical bond formed $\left(d_{1}\right)$. The bond lengths $d_{2}$, as shown in Fig. 2, decrease as the outer valence electron density of doped element increases from boron to oxygen. The resulting $\left(\mathrm{H}_{2}\right) \mathrm{O} \cdots \mathrm{H}^{+}$interaction pins water to the surface via weak H-bonds. This result may provide some hint to the observed hydrophobic or hydrophilic character of carbonbased materials treated in diverse aqueous solutions. In our cases, oxygen decorated edge is least hydrophobic. This is consistent with the experimental observation that on pristine graphene, which is hydrophobic but for which the measurement of the contact angle with water show that the wetting property can be improved by pre-adsorbed oxygen. ${ }^{39}$

Despite the shortest $\mathrm{H}$-bond distance $d_{2}$ on the oxygen-doped edge compared to others, which implies the highest $\mathrm{H}$-bonding strength, the relaxed interaction energy $\left(E_{i n t}\right)$ is the least exothermic. Hence, our results indicate that there exists an important balance between a hydronium ion's ability to form chemical-bonds and H-bonds on the edges. The stability of a hydronium at each edge is determined by the energy gain by forming the chemical and hydrogen bonds (edge $-\mathrm{H} \cdots \mathrm{OH}_{2}$ ). It is more advantageous to maximize the interaction energy caused by the covalent bonding between the edge atom and proton than to form the hydrogen bond. This could be the reason why the dissociated hydronium has the highest (least exothermic) energies when interacted with the oxygen doped edges.

\subsection{Mediating effect of water layer}

The present $a b$ initio calculations of the reactivity of a single hydronium with ribbon edges suggest the involvement of $\mathrm{H}$-bonding between either the hydronium and the edge atom $\left(r>r_{0}\right)$ or the dissociated water molecule and the proton $\left(r \leq r_{0}\right)$, as highlighted as dashed lines in Fig. 1. The question naturally arises whether $\mathrm{H}$-bonding interactions with the environmental water layers can change this balance and if there are some general cooperativity trends that can be established. In other words, is it possible that the bonding response of a single hydronium ion during charge carrier transfer to the edge may not accurately represent the response of a hydrogenated proton embedded in an aqueous environment? To explore this question, we have 
proceeded with extended calculations incorporating explicit surface water layers. This is a more demanding task from a computational point of view because many more electrons and relaxational degrees freedom need to be considered. While our exploration here is preliminary and would certainly support a fuller subsequent study, it provides useful insights nevertheless and hence we have included it in the present work.

In the mixed $\mathrm{H}_{3} \mathrm{O}^{+}+\mathrm{H}_{2} \mathrm{O}$ layer, water molecules are oriented based on the atomic structure of crystalline bulk ice. The ratio of single hydronium to water molecules numbers in examined model is one to eighteenth. The critical transition point defined by proton starting to transfer from hydrogenated charge carrier, i.e., hydronium to the active ribbon edge was studied in particular considering the mediated effect of water layer. We have done this by making qualitative analysis of relaxation geometries as the system is relaxed to the surface protonated state. Extensive real time molecular dynamics would also be desirable but is beyond the scope of the present study. The initial and relaxed geometries are shown in the upper and lower panels of Fig. 4, respectively. We find that the critical transition point of proton transfer including water layer effect was consistent with the case water-free when the exposed ribbon edge is $\mathrm{B}, \mathrm{C}$ or $\mathrm{N}$, in detail, the protons break from their hydronium group and move toward the edge of each ribbon, at respective critical distance $r_{0}$ obtained from previous study. In these examined cases, we did not observe a strong H-bonding effect of water molecules on the main features of hydronium dissociation. The hydrophobic domains are formed when the ribbon is exposed to water based on the fact that water molecules move away from the surface of ribbons during relaxation, and the water molecule deriving from the dissociated hydronium shifts in the same way. However, for oxygen-doped edge, proton is not specifically transferred. It appears that hydronium in the proximity of the oxygen-doped edge is stabilized due to the presence of nearby water molecules, leading to the detachment of hydronium ion in intact form away from the ribbon edge (Fig. 3), such that the hydronium does not specifically dissociate.

\subsection{Electronic structure features}

We first analyze the overall behavior of the electrostatic potential investigating its average value along the ribbon $z$ direction. Fig. 4 represents the average of the electrostatic potential (the sum of the Hartree and external potentials) for the ribbons. The position of the edge atom in pristine system is marked by a vertical dashed line in Fig. 4. The horizontal line in Fig. 4 corresponds to the electrostatic potential of pristine edge in vacuum $(3.26 \mathrm{eV})$. It clearly shows that deep inward from the edge the electrostatic potentials exhibit bulk-like behavior. Significant variations in the local electrostatic potential across the different doped edges are apparent. These are related to the reactivity of the unsaturated edge atoms, providing an alternative picture of how the electronic states at the bare edges react to charge carriers. The effective electrostatic potential of hydronium ion decreases rapidly as it approaches the edge from the vacuum. The hydronium ion is effectively reduced, decomposing into water and a chemically bonded proton with a certain degree of charge dispersal onto the ribbon. We have also recently discovered in a separate study that the ability to disperse additional positive

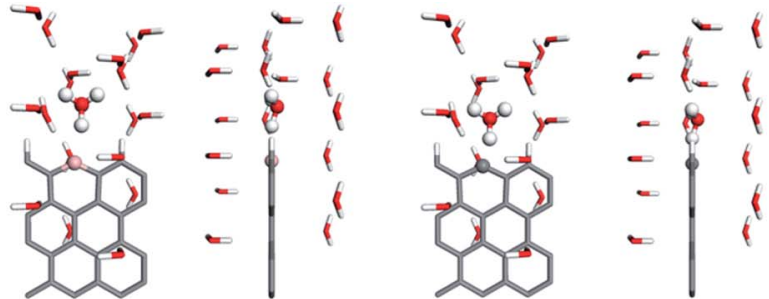

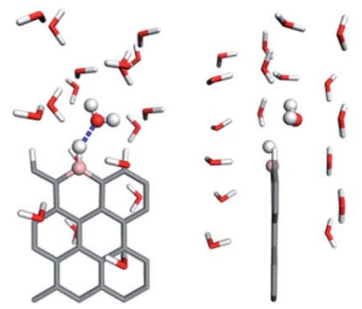

(a)
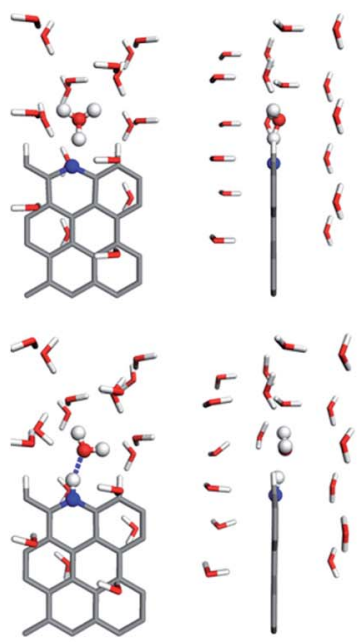

(c)

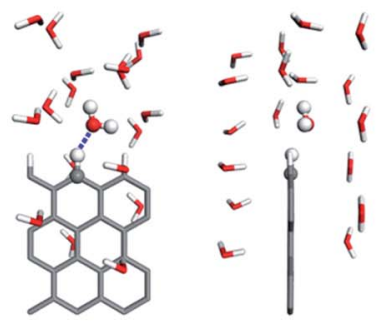

(b)
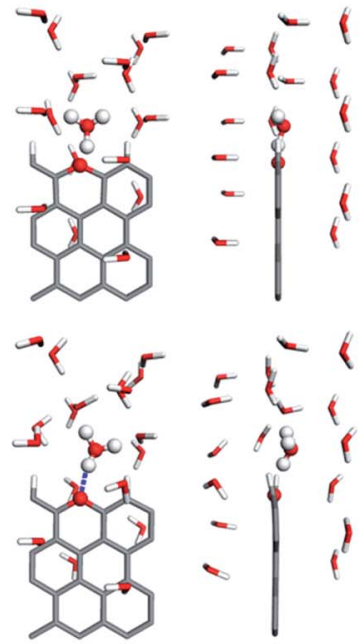

(d)
Fig. 3 Front and side views of the initial (upper panels) and relaxed (lower panels) structures of hydronium interacting with bare edge atoms of (a)-(d): B, C, N, and $\mathrm{O}$, respectively, with the inclusion of water molecules in the proximity of ribbon edge.

charge becomes a critical factor controlling the ability of different types of doped ribbons to load additional protons, specifically, the maximal loading of protons onto the substituted ZGNR edges obeys a rule of [8-n-1], where $n$ is the number of valence electrons of the edge-site atom constituting the adsorption site. ${ }^{40}$

When the proton is brought in from an "infinite" distance to bond with the edge atom, spatial rearrangement of electrons will occur accordingly at the contact interface. As such, charge carrier transfer between the proton carrier and ribbon edge is induced solely through their interaction. Calculated electron density difference (EDD) can be used to qualitatively estimate the relative number and flowing directions of charge carriers transferred between proton and the active site of doped ribbon edge upon their interaction. EDD is evaluated from the total charge density of the fully relaxed ribbon+hydronium 


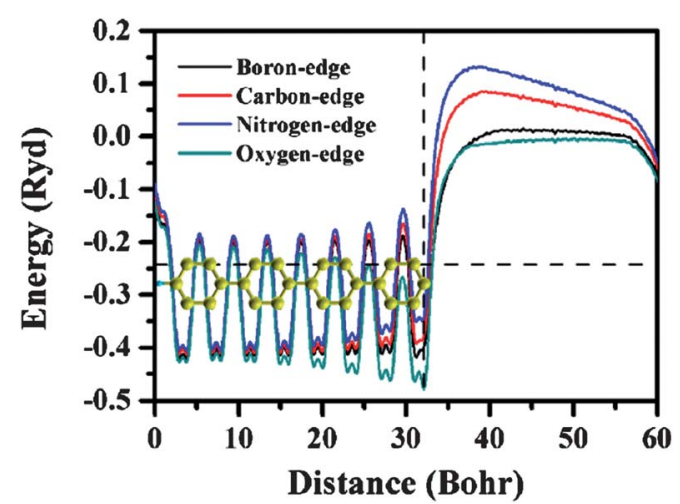

Fig. 4 Planar average of the electrostatic potential across the width direction of graphene nanoribbons with edges doped by (a) B, (c) N, (d) $\mathrm{O}$ and undoped (b). Potential line-up for the different doped ribbons done through pristine ribbon level. The dashed horizontal line represents the Fermi level of pristine system, while the dashed vertical line fixes the edge of pristine ribbon.

interaction system minus that of the system with ribbon and decomposed hydronium separately, as shown in Fig. 5.

The effective positive charge on the proton makes it a strong electron acceptor. This results in strong accumulation of electrons at chemically bonded proton site as observed in Fig. 5, which our DFT geometry optimizations show to be a general phenomenon for the proton/edge of all studied materials. Electron density difference also can be a useful tool to identify the hole transfer. Our calculated results suggests that holes will readily moves from proton to inner ribbon atoms as visualized as electron depletion. But the observable electron depletion is only shown when the edge carbon atom is replaced by dopants. Therefore, the principal proton transfer results in charge carrier rearrangement, hole inward and electron outward, and the formation of the contact ion pair. The charge carrier transfer observed in proton exchange becomes more complicated in when a strong hydrogen bond remains with the partner water molecule next to the edge site. According to our calculations, electron density transferred to the proton can, especially for $\mathrm{N}$ or $\mathrm{O}$ doped edge, be partially transferred to the dissociated water molecule. We can view the gain of electron in the $\mathrm{O}$ lone pair as the hydrogen atoms in water molecule becoming more positive. These rearrangements maximize the electrostatic attraction, allowing for the relatively short H-bond length. Mulliken population analysis was carried out to determine the amount of the

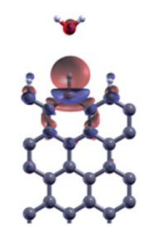

(a)

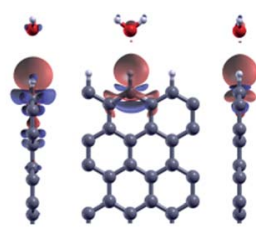

(b)

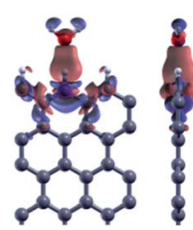

(c)

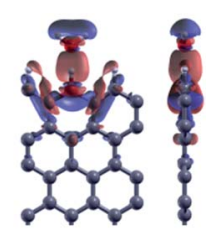

(d)
Fig. 5 Front and side views of the isosurfaces of total charge difference upon proton transferred from hydronium to the edge of doped ZGNRs with (a) boron, (b) pristine, (c) nitrogen, and (d) oxygen (isovalues for all the isosurfaces are $\pm 0.005 \mathrm{e}^{-3}$ ). Red indicates electronic density gain, blue indicates electronic density loss. transferring charge when proton was transferred from hydronium $\mathrm{H}_{3} \mathrm{O}^{+}$to the doped ribbon edge (Table $\mathrm{S} 1$ in ESI). Mulliken population based on the linear combination of atomic orbitals molecular orbital method provides a rough estimation on the partial atomic charges associated with each atom during the transferring process. It is noticeable that transferred proton on the B-doped edge site is characterized by large electron accumulation compared to the case before transferring, also to other doped cases, which conclusion is consistent with EDD analysis.

Charge carrier rearrangement is critical to charge transfer and collection at the electrode. Our results here indicate that doped ribbon edges may exhibit higher charge carrier conductivities and that this is a complex phenomenon requiring analysis from a number of different angles. A first principle quantum approach is a prerequisite to teasing apart this mechanistic picture. Indeed, not only edge-doped atoms but more generally functional groups that induce significant charge transfer could be used to enhance electrode design for the efficient collection of charge.

\section{Conclusions}

Using density functional theory we have examined mechanisms of charge transfer upon interaction of hydrated protons with a variety of doped carbon nanoribbons, both with and without an explicit water environment. As the hydronium ion approaches the exposed ribbon edge from a distance, it experiences a structural transition from simple H-bonded physical interaction to structural dissociation. The equilibrium configuration in the latter case ends in a chemically bonded proton to the edge and a weak hydrogen bond interaction between proton and dissociated water molecule for all cases except the O-doped nanoribbon. The contributions of hydrogen bonding and $\sigma$-based interaction to the overall interaction energies are evident.

The $\mathrm{B}, \mathrm{N}$, and $\mathrm{O}$ doped edges all show active proton affinity. The doped edges featuring dispersive orbital characteristics, i.e. boron and nitrogen atoms, are more energetically favourable for charge carrier exchange, compared to the oxygen dopant with more localized orbitals. Hence, heteroatom doping on the edge of graphene nanoribbons may provide tenability by changing the chemical activity of the edge with respect to charge carrier transfer in an aqueous environment. Analysis of the charge difference distribution reveals an obvious electrons outward mobility to the edge, while hole density moves conversely towards the inner side of ribbon.

\section{Acknowledgements}

TL acknowledges financial support from the University of Queensland Postdoctoral Research Fellowship. We also appreciate the generous grants of CPU time from both the University of Queensland and the Australian National Computational Infrastructure Facility. SCS acknowledges support from the Center for Nanophase Materials Sciences, which is sponsored at the Oak Ridge National Laboratory by the Scientific User Facilities Division, US Department of Energy.

\section{Notes and references}

1 C. Chattopadhyay, L. Chen and K. Chen, NPG Asia Mater., 2011, 3, 74. 
2 Z. Q. Sun, J. H. Kim, Y. Zhao, F. Bijiarbooneh, V. Malgras, Y. Lee, Y. M. Kang and S. X. Dou, J. Am. Chem. Soc., 2011, 133, 19314.

3 C. G. Liu, Z. N. Yu, D. Neff, A. Zhamu and Bor Z. Jang, Nano Lett., 2010, 10, 4863

4 R. Miller, R. A. Outlaw and B. C. Holloway, Science, 2010, 329, 1637.

5 M. D. Stroller, S. J. Park, Y. W. Zhu, J. H. An and R. S. Ruoff, Nano Lett., 2008, 8, 3498.

6 Y. W. Zhu, S. Murali, W. W. Cai, X. S. Li, J. W. Suk, J. R. Potts and R. S. Ruoff, Adv. Mater., 2010, 22, 3906.

7 C. Ataca, E. Aktürk, S. Ciraci and H. Ustunel, Appl. Phys. Lett., 2008, 93, 043123.

8 M. Wang and C. M. Li, Nanoscale, 2012, 4, 1044.

9 M. Wang, W. Huang, M. B. Chan-Park and C. M. Li, Nanotechnology, 2011, 22, 105702.

10 M. Wang and C. M. Li, Nanoscale, 2011, 3, 2324.

11 L. T. Qu, Y. Liu, J. B. Baek and L. M. Dai, ACS Nano, 2010, 4, 1321.

12 K. P. Loh, Q. L. Bao, P. K. Ang and J. X. Yang, J. Mater. Chem., 2010, 20, 2277.

13 L. H. Tang, Y. Wang, Y. M. Li, H. B. Feng, J. Lu and J. H. Li, $A d v$. Funct. Mater., 2009, 19, 2782.

14 M. Pumera, Chem. Rec., 2009, 9, 211

15 R. L. McCreery, Chem. Rev., 2008, 108, 2646.

16 H. Bai, Y. X. Xu, L. Zhao, C. Li and G. Q. Shi, Chem. Commun. 2009, 1667.

17 J. L. Johnson, A. Behnam, S. J. Pearton and A. Ural, Adv. Mater., 2010, 22, 4877.

18 R. Sharma, J. H. Baik, C. J. Perera and M. S. Strano, Nano Lett., 2010, 10, 398.

19 O. Hod, V. Barone, J. E. Peralta and G. E. Scuseria, Nano Lett., 2007, 7, 2295.

20 J. T. Sun, Y. H. Lu, W. Chen, Y. P. Feng and A. T. S. Wee, Phys. Rev B: Condens. Matter Mater. Phys., 2010, 81, 155403.

21 D. Hulicova-Jurcakova, M. Kodama, S. Shiraishi, H. Hatori, Z. H. Zhu and G. Q. Lu, Adv. Funct. Mater., 2009, 19, 1800.
22 B. F. Habenicht, S. J. Paddison and M. E. Tuckerman, J. Mater. Chem., 2010, 20, 6342.

23 K. N. Kudin and R. Car, J. Am. Chem. Soc., 2008, 130, 3915.

24 C. P. Chiu and C. W. Hong, J. Fuel Cell Sci. Technol., 2010, 7, 021003.

25 B. F. Habenicht, S. J. Paddison and M. E. Tuckerman, Phys. Chem. Chem. Phys., 2010, 12, 8728.

26 F. Wang, S. Izvekov and G. A. Voth, J. Am. Chem. Soc., 2008, 130, 3120.

27 S. V. Churakov and G. Kosakowski, Philos. Mag., 2010, 90, 2459.

28 S. Baroni, A. Dal Corso, S. de Gironcoli and P. Giannozzi, PWSCF (plane wave self consistent field) codes available at: http:// www.quantum-espresso.org.

29 J. P. Perdew, K. Burke and M. Ernzerhof, Phys. Rev. Lett., 1996, 77, 3865.

30 D. Vanderbilt, Phys. Rev. B: Condens. Matter, 1990, 41, 7892.

31 J. D. Pack and H. J. Monkhorst, Phys. Rev. B: Solid State, 1977, 16, 1748 .

32 N. Marzari and D. Vanderbilt, Phys. Rev. B: Condens. Matter, 1997, 56, 12847.

33 P. L. Silvestrelli, N. Marzari, D. Vanderbilt and M. Parrinello, Solid State Commun., 1998, 107, 7.

34 J. F. Dobson and B. P. Dinte, Phys. Rev. Lett., 1996, 76, 1780.

35 M. Dion, H. Rydberg, E. Schröder, D. C. Langreth and B. I. Lundqvist, Phys. Rev. Lett., 2004, 92, 246401.

36 U. Zimmerli, M. Parrinello and P. Koumoutsakos, J. Chem. Phys., $2004,120,2693$.

37 X. Wu, M. C. Vargas, S. Nayak, V. Lotrich and G. Scoles, J. Chem. Phys., 2001, 115, 8748.

38 D. W. Wang, F. Li, Z. G. Chen, G. Q. Lu and H. M. Cheng, Chem. Mater., 2008, 20, 7195.

39 S. R. Wang, Y. Zhang, N. Abidi and L. Cabrales, Langmuir, 2009, 25, 11078.

40 T. Liao, C. H. Sun, A. J. Du, Z. Q. Sun, D. H. Jurcakova and S. Smith, submitted to J. Mater. Chem. 